\title{
Il rene policistico autosomico dominante: l'importanza dello studio del volume renale
}

\section{R. Mignani, L. Cagnoli}

UOC di Nefrologia e Dialisi, Ospedale Infermi, Rimini

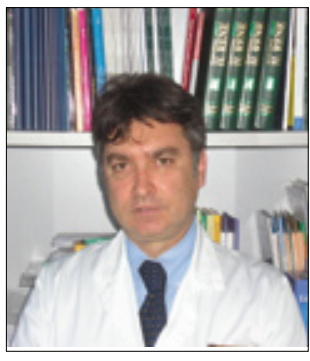

Renzo Mignani

\section{Introduzione}

Il rene policistico autosomico dominante dell'adulto (ADPKD) è una malattia genetica caratterizzata dalla comparsa di molteplici formazioni cistiche nel rene e in altri organi che evolve in oltre il $50 \%$ dei casi verso l'insufficienza renale cronica (IRC) terminale che richiede il trattamento sostitutivo con la dialisi o il trapianto.

L'ADPKD rappresenta la più frequente malattia renale da causa genetica manifestandosi con una incidenza variabile tra 1:800 e 1:1400 nati vivi e attualmente ne vengono riconosciute due varianti a seconda che la mutazione genetica sia localizzata nel cromosoma 16 (PKD1) o nel cromosoma 4 (PKD2) $(1,2)$. Le mutazioni del $P K D 1$ rappresentano l'85\% dei casi mentre il PKD2 rappresenterebbe il restante 15\% dei pazienti ADPKD. Pur localizzandosi in geni diversi, la malattia presenta un'unica espressione fenotipica caratterizzata dalla formazione di cisti a livello renale, epatico e in altri organi. Le due diverse alterazioni geniche PKD1 e $P K D 2$ codificano due diverse proteine transmembrana, la policistina 1 e la policistina 2 rispettivamente, entrambe espresse nell'epitelio tubulare renale e in molteplici altri tessuti (1-3). La produzione delle policistine 1 e 2 sarebbe alla base, secondo la teoria del cilio primario, delle alterazioni istomorfologiche che porterebbero alla formazione delle cisti. Normalmente le policistine sono localizzate sulla superficie del cilio primario, un organulo posto alla superficie delle cellule tubulari protrudente nel lume tubulare e funzionano come meccanorecettori che regolano l'in- gresso degli ioni Calcio nella cellula tubulare inibendo la proliferazione cellulare. Nei pazienti ADPKD le policistine inibiscono il passaggio del calcio intracellulare sopprimendo il meccanismo inibitorio sulla proliferazione cellulare con conseguente formazione di cisti (2). All'analisi istologica l'alterazione e la disorganizzazione strutturale della membrana basale delle cellule tubulari insieme alla proliferazione abnorme di tali cellule e alla apoptosi sarebbero all'origine della progressiva dilatazione dei tubuli e quindi alla formazione delle cisti che in seguito si separerebbero del tutto dal tubulo "madre". Nel frattempo, l'aumentata attività dell'c-AMP, stimolata dalla Vasopressina, presente nelle cellule dei pazienti con $\mathrm{ADPKD}$ oltre a stimolare anch'esso la proliferazione cellulare, aumenterebbe la produzione di fluido all'interno delle cisti renali favorendo la loro crescita $(4,5)$. Le policistine alterate inoltre possono stimolare la proliferazione cellulare attraverso la perdita di controllo della sintesi proteica mentre la policistina 1 interagisce con la $t u$ berina nel sopprimere il mediatore mTOR favorendo quindi la proliferazione cellulare (6).

Clinicamente la $\mathrm{ADPKD}$ si presenta in modo variabile: nei pazienti con $P K D 1$ la comparsa dei sintomi e la diagnosi sono precoci e avvengono attorno alla $2^{\mathrm{a}}-3^{\mathrm{a}}$ decade mentre nei pazienti con PKD 2 sia l'esordio che la diagnosi sono più tardivi avvenendo a volte anche alla $6^{\mathrm{a}}-7^{\mathrm{a}}$ decade di vita $(1,2)$. La sintomatologia d'esordio è rappresentata in genere dai dolori lombari o dalle coliche, dalla macroematuria e da infezioni delle vie urinarie. La diagnosi della malattia è in genere ecografica attraverso il riscontro di reni aumentati di volume e diffusamente occupati da formazioni cistiche. In alcuni casi il numero delle cisti renali è limitato per cui, soprattutto in assenza di familiarità, la 
diagnosi può essere difficile. A tale proposito, sono state di recente stabiliti i criteri diagnostici per porre diagnosi ecografica di rene policistico sia nel paziente con familiarità sia nei cosiddetti casi de novo senza familiarità nota per ADPKD (Tab. I) $(7,8)$. In alcuni casi i sintomi extrarenali della malattia possono precedere i sintomi renali. Così l'ipertensione può essere il primo sintomo in pazienti senza familiarità. In altri casi l'esordio può avvenire con una colica addominale dovuta alla compressione da parte di voluminose cisti epatiche. Gli aneurismi cerebrali si manifestano dal 5 al 16\% dei casi. La loro presenza è altamente condizionata dalla familiarità: nei pazienti con familiarità per aneurismi cerebrali e/o emorragie cerebrali l'incidenza degli aneurismi è del 16\% mentre nei pazienti senza familiarità è solo del 5\% (6). Altri sintomi sono legati alla presenza di difetti valvolari cardiaci, di ernie addominali e della diverticolosi del colon.

L'evoluzione dell'ADPKD è caratterizzata da un progressivo ingrandimento dei reni e delle cisti che si accompagna a una progressiva riduzione del parenchima renale funzionante per effetto della compressione da parte delle cisti. Tale processo sarebbe alla base della comparsa dell'IRC ma l'entità del parenchima renale residuo ancora funzionante al quale farebbe seguito la comparsa dell'IRC non è nota. Nei pazienti con $P K D 1$ la comparsa dell'IRC e l'inizio della dialisi è precoce e avviene attorno alla $3^{\mathrm{a}}-4^{\mathrm{a}}$ decade mentre nei pazienti con $P K D 2$ la progressione della malattia è più lenta e l'inizio della dialisi avviene non prima della $6^{\mathrm{a}}-7^{\mathrm{a}}$ decade. La progressione della $\mathrm{ADPKD}$ verso la IRC terminale è condizionata da diversi fattori quali l'ipertensione, l'età, il sesso e il volume dei reni e delle cisti.

Tra questi fattori la volumetria renale ha assunto di recente un ruolo rilevante nella progressione del danno renale nella $\mathrm{ADPKD}$. E ampiamente dimostrato come con l'aumentare del volume renale totale (VRT) il paziente tenda a manifestare una insufficienza renale ingravescente. Pertanto, nei pazienti con ADPKD l'entità della volume renale si può considerare come un indicatore prognostico dell'evoluzione della malattia policistica e quindi un marker surrogato di progressione dell'ADPKD. Pertanto, la riduzione del VRT potrà essere di grande beneficio non solo impedendo che i reni diventino di enormi dimensioni cosi da determinare complicanze cliniche tali da procedere alla nefrectomia per compressione sugli organi addominali e toracici e limitando il "danno estetico" conseguente all'ingrandimento dell'addome. Ma soprattutto, rallentare la crescita dei reni potrebbe in futuro impedire o al massimo ridurre la progressione della malattia verso l'IRC.

TABELLA I - EVOLUZIONE DEI CRITERI PER LA DIAGNOSI ECOGRAFICA DEL RENE POLICISTICO NELLE DIVERSE FASCE D’ETÀ

\begin{tabular}{|c|c|c|c|}
\hline $\begin{array}{l}\text { Età } \\
\text { (anni) }\end{array}$ & Criteri & $\begin{array}{c}\text { PPV } \\
\text { valore predittivo } \\
\text { positivo }\end{array}$ & $\begin{array}{c}\text { NPV } \\
\text { valore predittivo } \\
\text { negativo }\end{array}$ \\
\hline & Criteri diagnostici originali (Ravine) & & \\
\hline $15-29$ & $>2$ cisti, monolaterale o bilaterale & 99.2 & 87.7 \\
\hline $30-39$ & $>2$ cisti in ciascun rene & 100 & 87.5 \\
\hline $40-59$ & $>2$ cisti in ciascun rene & 100 & 94.8 \\
\hline \multirow[t]{2}{*}{$>60$} & $>4$ cisti in ciascun rene & 100 & 100 \\
\hline & Criteri diagnostici revisionati (Pei) & & \\
\hline $15-29$ & $>3$ cisti, monolaterale o bilaterale & 100 & 85.5 \\
\hline $30-39$ & $>3$ cisti monolaterale o bilaterale & 100 & 96.4 \\
\hline $40-59$ & $>2$ cisti in ciascun rene & 100 & 94.8 \\
\hline \multirow[t]{2}{*}{$>60$} & $>4$ cisti in ciascun rene & 100 & 100 \\
\hline & Criteri revisionati per escludere la di & & \\
\hline $15-29$ & $>1$ cisti & 96.6 & 90.8 \\
\hline $30-39$ & $>1$ cisti & 94 & 98.3 \\
\hline $40-59$ & $>2$ cisti & 96.7 & 100 \\
\hline$>60$ & $>4$ cisti in ciascun rene & 100 & 100 \\
\hline
\end{tabular}


TABELLA II - FATTORI IMPLICATI NELLA PROGRESSIONE DELLA MALATTIA POLICISTICA DEI RENI

\begin{tabular}{|c|c|c|}
\hline Variabile & Significatività & Commento \\
\hline Sesso & $\mathrm{p}<0.001$ & $\begin{array}{l}\text { I maschi hanno una funzione renale } \\
\text { peggiore delle femmine }\end{array}$ \\
\hline Genotipo & $\mathrm{p}<0.001$ & $\begin{array}{c}\text { Il genotipo } P K D 1 \text { ha una funzione renale } \\
\text { peggiore del } P K D 2\end{array}$ \\
\hline Età alla diagnosi & $\mathrm{p}<0.001$ & $\begin{array}{l}\text { I soggetti diagnosticati più precocemente } \\
\text { hanno una progressione maggiore della malattia } \\
\text { verso l'insufficienza renale }\end{array}$ \\
\hline Ipertensione & $\mathrm{p}<0.001$ & \\
\hline Dolore lombare & NS & \\
\hline Aneurismi cerebrali & NS & \\
\hline Cisti epatiche & $\mathrm{p}<0.001$ & Significativo solo nelle donne \\
\hline Episodi di macroematuria & $\mathrm{p}<0.01$ & \\
\hline Infezioni vie urinarie & NS & \\
\hline Volume Renale Totale & $\mathrm{p}<0.001$ & $\begin{array}{l}\text { Pazienti con volume renale maggiore hanno una } \\
\text { progressione dell'insufficienza renale più rapida }\end{array}$ \\
\hline Volume Totale Cisti & $\mathrm{p}<0.001$ & $\begin{array}{l}\text { Pazienti con volumi più elevati hanno una } \\
\text { progressione più rapida }\end{array}$ \\
\hline
\end{tabular}

NS: non significativo; $\mathrm{p}<0,001$ e $\mathrm{p}<0 ., 01$ : significativi.

(modificata da: P. Gabow et al. Kidney Int 1992; 41: 1311-9)

\section{I markers di progressione della malattia policistica}

Diversi sono i fattori responsabili della progressione dell'ADPKD (Tab. II).

L'età all'esordio della $\mathrm{ADPKD}$ ha mostrato una certa variabilità: spesso l'esordio clinico in età giovanile si accompagna a una più rapida evoluzione della malattia ma sono frequenti anche casi opposti in cui esordi clinici precoci mostrano una lenta evoluzione. In entrambi i casi sembra che ciò sia indipendente dal genotipo PKD1 o PKD2 del paziente. Anche il sesso potrebbe influenzare la prognosi poiché la malattia sembra esprimersi più tardivamente e più lentamente nelle femmine(9).

Il genotipo è un altro importante fattore in grado di influenzare la progressione della malattia: $\mathrm{i}$ pazienti $P K D 1$ hanno una evoluzione più rapida rispetto ai genotipi $P K D 2$. In che modo il genotipo possa influenzare la prognosi non è noto. Non sembra che possa influire né il tipo di mutazione né la posizione della stessa nel gene. È possibile invece che ciò sia dovuta alla maggior quantità di cisti presenti nel paziente $P K D 1$ e quindi a volumi renali più elevati in questi pazienti (10). Del resto, è ampiamente documentata una notevole eterogeneità intrafamiliare del fenotipo. Infatti è stato osservato come nei pazienti $P K D$ la malattia sembra evolvere più rapidamente verso la IRC terminale se il gene è ereditato dalle madri piuttosto che dai padri come a indicare una prognosi sfavorevole della trasmissione materna (11). Infine, un altro aspetto legato al genotipo sarebbe quello che sta alla base della teoria del "doppio insulto" nella genesi delle cisti e nella progressione della malattia. Secondo tale teoria, oltre al gene alterato PKD 1 o $P K D 2$ sarebbe necessaria la presenza di una ulteriore mutazione somatica per dare avvio al processo di formazione delle cisti (12).

L'effetto della macroematuria sulla progressione dell'ADPKD è controverso. In studi recenti, pazienti con frequenti episodi di macroematuria hanno presentato una evoluzione verso l'insufficienza renale più rapida rispetto ai pazienti senza una storia di ematuria. Ciò è stato attribuito al maggior numero e dimensioni delle cisti e quindi al volume renale più elevato documentato nei pazienti che vanno più spesso incontro alla macroematuria. Analogamente, i pazienti con più frequenti episodi di infezione delle vie urinarie andrebbero incontro a una evoluzione più rapida della malattia (3).

La proteinuria è presente solo nel 20\% dei pazienti con $\mathrm{ADPKD}$ e non supera in genere i $300 \mathrm{mg} / \mathrm{die}$. Con il peggiorare della funzione renale la proteinuria tende ad aumentare senza superare comunque il grammo/ 
die. Tuttavia, la proteinuria sembra la conseguenza della progressione più che la causa della IRC come invece avviene nelle glomerulonefriti. Al contrario, la proteinuria nei pazienti $\mathrm{ADPKD}$ sembra condizionata anch'essa dagli elevati volumi renali essendo più frequente e aumentando maggiormente nei pazienti con elevati valori di VRT (13).

Lipertensione arteriosa è un frequente sintomo dell'ADPKD e riveste un ruolo importante nella progressione della malattia oltre che nella morbidità cardiovascolare. Il fatto che la comparsa dell'ipertensione avvenga in oltre il $75 \%$ dei pazienti ADPKD prima della dell'insufficienza renale e che una ipertensione sia documentabile anche nei bambini affetti da ADPKD dell'adulto, ha portato alla conclusione che è più probabile che l'ipertensione contribuisca al peggioramento della funzione renale piuttosto che il danno renale dovuto alla presenza di cisti sia responsabile dell'ipertensione stessa. Pertanto, è fortemente raccomandata l'introduzione della terapia anti-ipertensiva anche nei bambini con ipertensione borderline (14). Tra i fattori responsabili dell'ipertensione l'attivazione del sistema renina-angiotensina (SRA) gioca senza dubbio un importante ruolo ed è probabilmente dovuto all'ischemia del microcircolo renale indotta dall'espansione delle cisti. Del resto l'ipertensione è strettamente correlata al volume renale: numerosi studi hanno infatti dimostrato che VRT è significativamente più elevato nei pazienti ipertesi piuttosto che nei normotesi con $\mathrm{ADPKD}(9,15,16)$.

Anche la presenza delle cisti epatiche, documentata nel 70-90\% dei casi, è importante nella progressione dell'ADPKD. La loro presenza è rarissima nei bambini, aumenta con l'età in entrambi i genotipi PKD1 e $P K D 2$ ed è maggiore nelle femmine che nei maschi (17). Raramente il fegato policistico evolve verso la insufficienza epatica e altrettanto raramente quando particolarmente sintomatiche richiedono lo svuotamento con agoaspirazione percutanea.

La progressione dell'ADPKD verso la fase terminale dell'IRC è molto variabile e il meccanismo dell'insufficienza renale non è ancora del tutto chiaro. Nei pazienti con $\mathrm{ADPKD}$ la funzione renale può rimanere normale per anni nonostante la presenza di cisti renali diffuse. Al contrario, una IRC è stata descritta anche nei bambini (18). Il genotipo sembrerebbe avere un ruolo importante sull'epoca di comparsa dell'IRC ma non sulla possibilità di andare incontro o meno alla formazione di cisti renali, cosa che avviene sempre una volta ereditato il gene (19): nel PKD2 infatti l'IRC comparirebbe almeno 15 anni dopo che nel PKD2 (9, 20). Quindi, nonostante tutti gli individui che hanno ereditato il $P K D 1$ o il $P K D 2$ sviluppino cisti renali, non tutti progrediscono verso l'IRC terminale. Circa poi la velocità di progressione dell'IRC, in uno studio eseguito su 141 pazienti con ADPKD e lieve IRC la perdita di funzione renale calcolata con la formula del Glomerular Filtration Rate (GFR) era di 5,9 ml/ min/anno (21). Tra le cause che farebbero evolvere l'ADPKD verso l'IRC il volume delle cisti e quindi del rene viene oramai considerato tra le più importanti. Sin dagli studi di Dalgaard, eseguiti in un epoca in cui non esisteva l'ecografia, è noto come il volume dell'addome e quindi le dimensioni delle masse renali alla palpazione dell'addome correlino in modo inverso con la funzionalità renale (22). In seguito molteplici studi in cui il volume renale veniva misurato con diverse tecniche radiologiche hanno confermato la tesi di Dalgaard. Pertanto, l'opinione che sempre più sta emergendo è che il volume renale rappresenti forse il principale fattore predittivo e prognostico sulla evoluzione della malattia policistico verso l'IRC.

\section{Lo studio della volumetria renale}

Lo studio del volume renale nell'ADPKD ha assunto in quest'ultimi anni un ruolo decisivo determinando una notevole accelerazione nella conoscenza della malattia e attualmente si può affermare che la velocità con cui il rene aumenta il suo volume rappresenti la misura della velocità di progressione della malattia stessa (23-25). I medici hanno quindi il dovere di informare i pazienti di quanto sono cresciuti i loro reni e dei rivolgimenti che una progressiva crescita possa determinare sulla sintomatologie e sulla progressione della loro malattia. Molte cisti si formano già in utero ma la maggior parte inizia a svilupparsi in epoca post-natale. Tuttavia, le cisti che compaiono in utero hanno una crescita più rapida delle cisti postnatali. Nell'adulto, studi recenti basati sull'impiego della RM hanno dimostrato come l'aumento del volume renale sia un processo rapido avvenendo già in modo significativo dopo solo 6 mesi dal controllo basale (26).

E oramai noto che esiste una correlazione diretta tra VRT e VTC, per cui se aumentano le cisti aumentano di conseguenza anche i reni e per tale motivo si fa riferimento in genere al volume in toto dei reni anche perché la misurazione del volume delle cisti spesso è difficile (27). Con l'aumentare del volume i reni mantengono quasi del tutto la loro morfologia ma il peso aumenta sino a oltre $4 \mathrm{~kg}$ ciascuno (il peso di un rene normale è di 120-140 grammi). I pazienti che mostra- 
no una rapida crescita del volume dei reni e delle cisti sono a rischio di un più rapido peggioramento della funzione renale rispetto ai pazienti con basso volume renale al momento della diagnosi (6).

La misura del volume renale viene attualmente ottenuta con tecniche radiologiche di imaging come la tomografia computerizzata (TC) e la risonanza magnetica (RM) che si sono dimostrate le migliori per affidabilità e riproducibilità $(28,29)$. Sia la TC che la $\mathrm{RM}$ sono in grado di delineare i contorni renali, di calcolarne il volume, di individuare le caratteristiche morfologiche delle cisti, la loro distribuzione nel parenchima, la loro simmetria rivelando variazioni entro brevi intervalli di tempo. La TC ha però l'inconveniente di basarsi sull'emissione di radiazioni ionizzanti e di usare il mezzo di contrasto iodato nefrotossico nei pazienti con IRC. La RM sembra invece l'indagine migliore perché è in grado di ottenere immagini tridimensionali ad alta risoluzione senza esporre il paziente alla radiazioni ionizzanti e a mdc iodinati. Entrambi le acquisizioni T-1 e T-2 pesate sono in grado di caratterizzare in modo ottimale i contorni renali ottenuti con la tecnica stereologica manuale (30). L'impiego del gadolinio tuttavia, necessario per ottimizzare le immagini delle cisti e dei contorni, presenta una forte limitazione quando deve essere impiegato nei pazienti con IRC avanzata per la possibilità di indurre la Fibrosi Sistemica Nefrogenica. Tale complicanza è caratterizzata da un interessamento fibrotico della cute che presto si diffonde ad altri organi. Attualmente si sconsiglia l'impiego di tale sostanza nei pazienti con deficit funzionale renale avanzato pari a un GFR $<60 \mathrm{ml} / \mathrm{min}$ (31). Inoltre il gadolinio avrebbe anche un effetto di "rigonfiamento" dei reni poiché è dimostrato che il VRT prima dell'infusione di tale sostanza è significativamente inferiore al VRT calcolato dopo la somministrazione (32).

La misura del volume delle cisti è più difficile della misura del volume renale. Le cisti infatti spesso sono di piccolissime dimensioni e hanno diverse caratteristiche di segnale. Ad esempio le cisti "complicate" a contenuto emorragico hanno caratteristiche di segnale diverso dalle cisti non complicate (33). Inoltre le cisti poste in sede corticale sono spesso adiacenti al fegato o alla milza rendendo difficile la delineazione del contorno renale. Per ragioni non conosciute la crescita dei reni è spesso asimmetrica cosicché il volume di un rene aumenta più rapidamente del controlaterale. Ciò si verifica perché le cisti di quel rene crescono di numero e di dimensioni maggiormente che nell'altro. I pazienti con $P K D 1$ hanno un numero di cisti significativamente superiore ai pazienti con $P K D 2$ mentre la

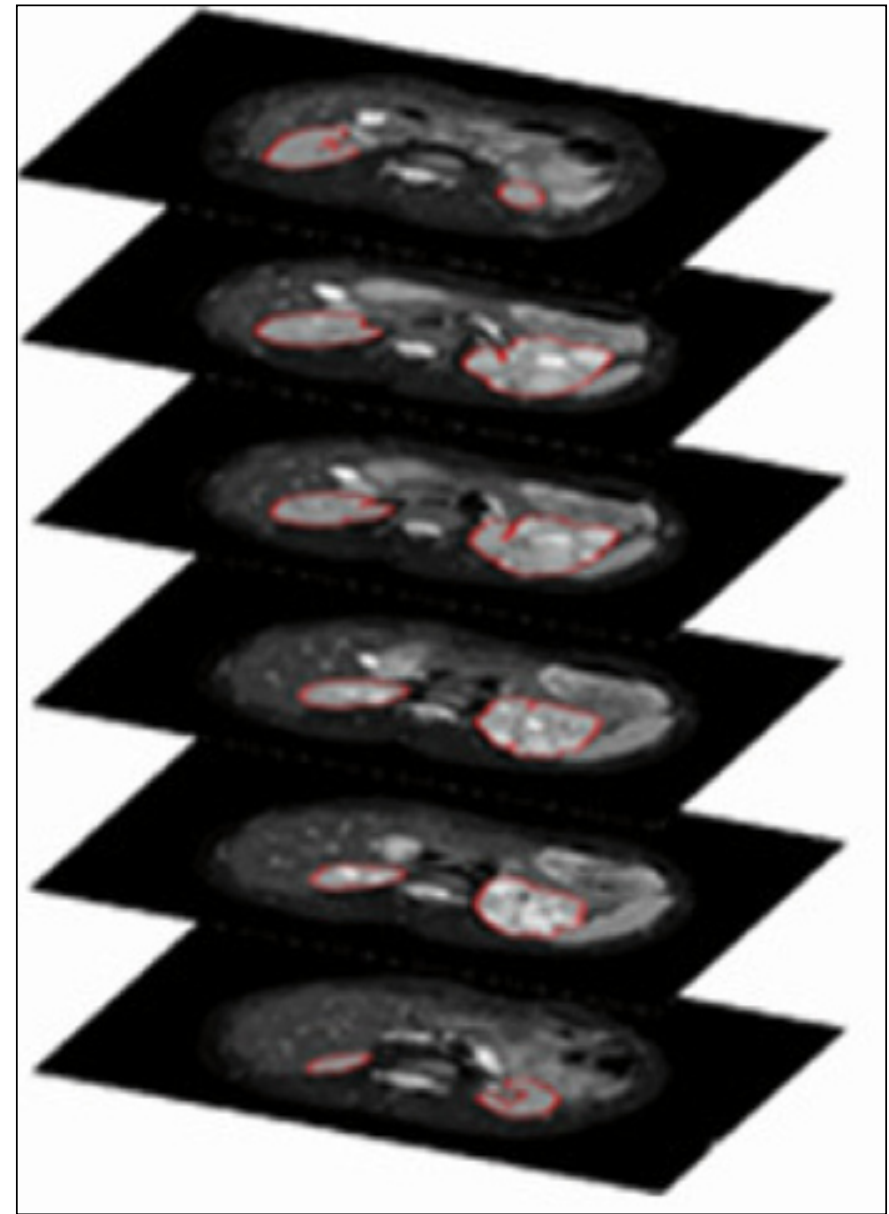

Figura 1 - Esempio di tracciamento automatico dei contorni delle sezioni dei reni policistici ottenute con $R M$ senza mezzo di contrasto: partendo da un punto posto all'interno del parenchima renale si ottiene automaticamente il contorno renale di una sezione che sommato alle sezioni sovra e sottostanti consente di ottenere la stima del volume renale totale ( $\mathrm{da}$ Corsi $\mathrm{C}$. et $\mathrm{al}, 51^{\circ} \mathrm{Con}$ vegno della Società Italiana di Nefrologia, Bologna 2009).

velocità di crescita delle stesse sembra la medesima nei due diversi genotipi (10).

Di recente, uno studio tutto italiano ha valutato il VRT con l'impiego della RM senza mdc e con la misura automatica del VRT. In questo studio eseguito su 30 pazienti con ADPKD di cui 8 con IRC avanzata l'analisi RM veniva eseguita con acquisizioni T1 e T2 pesate. L'automatismo è risultato il vero aspetto innovativo rispetto alla misura tradizionale stereologica con tracciamento manuale del contorno renale. Con tale tecnica, il tempo di calcolo del volume renale si reduce a soli 3 minuti, contro i 25-30 minuti della tecnica non automatica stereologica escludendo quasi totalmente l'intervento dell'operatore (Fig. 1). La tecnica si è rivelata sicura, 
rapida e affidabile con un errore di stima di soli $1,9 \%$ rispetto alla tecnica stereologica tradizionale e ha consentito la misura del volume anche in pazienti con IRC avanzata non avendo impiegato il gadolinio (34).

Molteplici studi hanno dimostrato come la velocità di peggioramento della funzione renale correli con il ritmo di crescita del volume renale. Nei maschi questa correlazione è risultata più evidente che nelle femmine a parità di età. Se poi i pazienti venivano suddivisi in base all'entità del VRT iniziale è stato dimostrato che il peggioramento della funzione renale era maggiore nel gruppo di pazienti con VRT al baseline $>1500 \mathrm{ml}$ rispetto sia al gruppo di pazienti con VRT inferiore $(<750$ $\mathrm{ml}$ ) sia rispetto ai pazienti con VRT al baseline tra 750-1500 ml (27).

\section{Gli effetti dei farmaci sulla volume- tria renale}

Sulla base del presupposto che probabilmente il VRT rappresenta il principale fattore prognostico nella progressione dell'ADPKD verso la IRC, sono stati condotti numerosi studi sull'impiego di farmaci capaci di rallentare la crescita dei reni basati sull'impiego di tecniche radiologiche di imaging per calcolare le variazioni di volume.

Uno dei primi studi è stato condotto in Italia su 12 pazienti con $\mathrm{ADPKD}$ e normale funzione renale sottoposti a TC con mdc prima e dopo 6 mesi di trattamento con Somatostatina, una farmaco in grado di inibire la generazione del c-AMP indotta dalla Vasopressina. Al termine del periodo di studio i pazienti mostravano una crescita del volume renale significativamente inferiore rispetto ai pazienti trattati con placebo (35). Lo stesso gruppo nefrologico, in uno studio controllato eseguito su 10 pazienti con $\mathrm{ADPKD}$ e funzione renale normale o lievemente compromessa trattati con l'inibitore m-TOR Sirolimus, osservava dopo 6 mesi di trattamento una ridotta crescita dei reni studiata con la TC nei pazienti trattati con Sirolimus rispetto ai pazienti in terapia convenzionale, mentre non si osservava alcun beneficio del farmaco sulla funzione renale (36). Più di recente tuttavia, uno studio controllato, randomizzato verso placebo ha valutato l'efficacia del Sirolimus in 100 pazienti con $\mathrm{ADPKD}$ e normale funzione renale. Dopo 18 mesi di trattamento il VRT studiato con RM non diminuiva nei pazienti trattati con Sirolimus e la funzione renale rimaneva stabile (37). Un altro studio basato invece sull'impiego di Everolimus ha mostrato risultati analogamente deludenti non essendosi osservata una diminuzione significativa del volume renale e del GFR in oltre 400 pazienti ADPKD dopo 24 mesi di trattamento (38). I deludenti risultati di tali studi in termini di rallentamento della progressione dell'IRC, sono stati oggetto di un'ampia discussione da parte della comunità scientifica che tuttora non ha consentito di confermare in modo definitivo l'assenza di efficacia e quindi di escludere in futuro l'impiego di tale categoria di farmaci nell'ADPKD $(39,40)$. Altri studi in corso sono basati sull'impiego di sostanze come il Tolvaptan in grado di inibire il c-AMP con il fine di rallentare la progressione della malattia attraverso la riduzione del volume renale. Uno studio recente e preliminare ha dimostrato una significativa riduzione del volume dei reni e delle cisti in un piccolo gruppo di pazienti $\mathrm{ADPKD}$ trattati per un breve periodo con tale farmaco(41).

\section{Conclusioni}

L'aumento del volume renale è oramai considerato come il fattore più importante nel condizionare il peggioramento della funzione renale nei pazienti ADPKD. Pertanto la misurazione del volume renale ha assunto una valenza prognostica sulla malattia. Tra le varie tecniche radiologiche, la TC e la $\mathrm{RM}$ rappresentano le più appropriate per la determinazione del volume renale.

I risultati preliminari basati sull'impiego sperimentale di farmaci in grado di ridurre la crescita dei reni con l'intento di ridurre la progressione della malattia sono al momento contrastanti e accanto a studi promettenti, altri più recenti si sono dimostrati apparentemente deludenti nel dimostrare un arresto della progressione della malattia. Tuttavia, diverse obiezioni sono state poste alla metodologia applicata a tali studi come la possibile insufficienza delle dosi dei farmaci somministrati, i livelli ematici dei farmaci bassi e insufficienti, il breve periodo di studio. Pertanto al momento una prova convincente dell'efficacia di tali sostanze nel ridurre la progressione della malattia verso l'insufficienza renale non c'è ma si auspica che il perfezionamento e il prolungamento di tali studi possa in futuro portare a risultati più eclatanti che possano portare all'impiego di tali farmaci a tutti i pazienti con $\mathrm{ADPKD}$. 
Indirizzo degli Autori:

Dr. Renzo Mignani

UOC Nefrologia e Dialisi

Ospedale Infermi

Via Settembrini 2

47900 Rimini

rmignani@auslrn.net

\section{Bibliografia}

1. Gabow P. Autosomal dominant polycystic kidney disease. N Engl J Med 1993; 329:332-342.

2. Grantham JJ. Clinical practice. Autosomal dominant polycystic kidney disease. New Engl J Med 2008; 359: 14771485.

3. Gabow PA, Duley I, Johnson AM. Clinical profiles of gross hematuria in autosomal dominant polycystic kidney disease. Am J Kidney Dis 1992; 20: 140-143.

4. Nauli S, Alenghart FJ, Luo Y. Polycystins 1 and 2 mediate mechanosensation in the primary cilium of kidney cells. Nat Gen 2003;33: 129-137 .

5. Grantham JJ Flu id secretion, cellular proliferation and the pathogenesis of renal epithelial cysts. J Am Soc Nephrol 1993; 3: 1841-1857.

6. Torres VE, Harris PC. Autosomal dominant polycystic kidney disease: the last 3 years. Kidney International 2009; 76: 149-168.

7. Ravine D, Gibson RN, Walker RG, Sheffield LJ, KincaidSmith P, Danks DM. Evaluation of ultrasonographic diagnostic criteria for autosomal dominant polycystic kidney disease 1. Lancet 1994; 343: 824-827.

8. Pei Y, Obaji J, Dupuis A, Paterson AD, Magistroni R, Dicks E, Parfrey P, Cramer B, Coto E, Torra R, San Millan JL, Gibson R, Breuning M, Peters D, Ravine D. Unified Criteria for Ultrasonographic Diagnosis of ADPKD. J Am Soc Nephrol 2009; 20: 205-212.

9. Gabow PA, Johnson AM, Kaehny WD, Kimberling WJ, Lezotte DC, Duley IY, Jones RH. Factors affecting the progression of renal disease in autosomal dominant polycystic kidney disease. Kidney Int 1992; 41: 1311-1319.

10. Harris PC, Bae KT, Rossetti S, Torres VE, Grantham JJ, Arlene B. Chapman AB, Guay-Woodford LM, King BF, Wetzel LH, Baumgarten DA, Kenney PJ, Consugar M, Klahr S, Bennett WM, Meyers CM, Zhang Q, Thompson PA, Zhu F, Miller JP and the CRISP: Cyst Number but Not the Rate of Cystic Growth Is Associated with the Mutated Gene in Autosomal Dominant Polycystic Kidney Disease. J Am Soc Nephrol 2006; 17: 3013-3019.
11. Bear JC, Parfrey PS, Morgan JM, Martin CJ, Cramer BC. Autosomal dominant polycystic kidney disease: New information for genetic counselling. Am J Med Genet 1992: 43:548-553.

12. Pei Y. A "two-hit" model of cystogenesis in autosomal dominant polycystic kidney disease? Trends Mol Med 2001 Apr;7(4):151-6.

13. Chapman AB, Johnson AM, Gabow PA, Schnien RW: Overt proteinuria and microalbuminurla in autosomal dominant polycystic kidney disease. J Am Soc Nephrol 1994;5: 1349-1354.

14. Cadnapaphornchai MA, McFann K, Strain JD, Masoumi A, Schrier RW. Increased left ventricular mass in children with autosomal dominant polycystic kidney disease and borderline hypertension. Kidney Int 2008; 74: 1192-1196.

15. Chapman AB, Guay-Woodford LM, Grantham JJ, Torres VR, Bae KT, Baumgarten DA, Kenney PJ, King BF, jr., Glockner JF, Wetzel LH, Brummer ME, O'Neill WC, Robbin MI, Bennett WM, Klahr S, Hirschman GH, Kimmel PI, Thompson PA, and Miller JP. Renal structure in early autosomal-dominant polycystic kidney disease (ADPKD): The Consortium for Radiologic Imaging Studies of Polycystic Kidney Disease (CRISP) cohort. Kidney Int 2003; 64: 1035-1045.

16. Lee YR, Lee KB. Reliability of Magnetic Resonance Imaging for Measuring the Volumetric Indices in AutosomalDominant Polycystic Kidney Disease: Correlation with Hypertension and Renal Function. Nephron Clin Pract 2006; 103:c173-c180.

17. Bae KT, Zhu F, Chapman AB, Torres VE, Grantham JJ, Guay-Woodford LM, Baumgarten DA, King BF, Jr., Wetzel LH, Kenney PJ, Brummer ME, Bennett WM, Klahr S, Meyers CM, Zhang X, Thompson PA, Miller JP and the Consortium for Radiologic Imaging Studies of Polycystic Kidney Disease (CRISP). Magnetic Resonance Imaging Evaluation of Hepatic Cysts in Early Autosomal-Dominant Polycystic Kidney Disease: Polycystic Kidney Disease Cohort. Clin J Am Soc Nephrol 2006; 1: 64-69.

18. Sedman A, Bell P, Manco-Johnson M, Schrier R, Warady BA, Heard EO, Butler-Simon N, Gabow P. Autosomal dominant polycystic kidney disease in childhood: A longitudinal study. Kidney Int 1987;31: 1000-1005.

19. Grantham JJ, Chapman AB, Torres VE. Volume progression in autosomal dominant polycystic kidney disease: The major factor determining clinical outcomes: Clin J Am Soc Nephrol 2006;1: 148-157.

20. Magistroni R, He N, Wang K, Andrew R, Johnson A, Gabow P, Dicks E, Parfrey P, Torra R, San-Millan JL, Coto E, Van Dijk M, Breuning M, Peters D, Bogdanova N, Ligabue G, Albertazzi A, Hateboer N, Demetriou K, Pierides A, Deltas C, St George-Hyslop P, Ravine D, Pei Y. Genotype-renal function correlation in type 2 autosomal 
dominant polycystic kidney disease. J Am Soc Nephrol 2003;14: 1164-1174.

21. Choukroun G, ItakuraY, Albouze G, Christophe JL, Man NK, Grunfeld JP, Jungers P. Factors Influencing Progression of Renal Failure in Autosomal Dominant Polycystic Kidney Disease. J Am Soc Nephrol 1995; 6:1634-1642.

22. Dalgaard OZ. Bilateral polycystic disease of the kidneys. Acta Med Scand 1957; 328: 1-255.

23. King BF, Reed JE, Bergstralh EJ, Sheedy PF 2nd, Torres VE. Quantification and longitudinal trends of kidney, renal cyst, and renal parenchyma volumes in autosomal dominant polycystic kidney disease: J Am Soc Nephrol 2000; 11: 1505-1511.

24. Perrone R. Imaging Progression in Polycystic Kidney Disease. N Engl J Med 2006; 354: 2181-2183.

25. Bae KT and Grantham JJ. Imaging for the prognosis of autosomal dominant polycystic kidney disease. Nat Rev Nephrol 2010; 6: 96-106.

26. Kistler AD, Poster D, Krauer F, Weishaupt D, Raina S, Senn O, Binet I, Spanaus K, Wuthrich RP and Serra AL: Increases in kidney volume in autosomal dominant polycystic kidney disease can be detected within 6 months. Kidney Int 2009; 75: 235-241.

27. Grantham J J , Vicente E. Torres VE, Chapman AB, GuayWoodford LM, Bae KT, King BF, Wetzel LH, Baumgarten DA, Kenney PJ, Harris PC, Klahr S, Bennett WM, Hirschman GN, Meyers CM, Zhang X, Zhu F, MillerJP for the CRISP Investigators. Volume progression in polycystic kidney disease. N Engl J Med 2006; 354: 2122-2130.

28. Sise C, Kusaka M, Wetzel LH, Winklhofer F, Cowley BD, Cook LT, Gordon M, Grantham JJ. Volumetric determination of progression in autosomal dominant polycystic kidney disease by computed tomography. Kidney Int 2000; 58: 2492-2501.

29. Antiga L, Piccinelli M, Fasolini G, Ene-Iordache B, Ondei P, Bruno S, Remuzzi G, Remuzzi A. Computed Tomography Evaluation of Autosomal Dominant Polycystic Kidney Disease Progression: A Progress Report. Clin J Am Soc Nephrol 2006; 1: 754-760.

30. Bae KT, Commean PK, Lee J. Volumetric measurement of renal cysts and parenchyma using MRI: phantoms and patients with polycystic kidney disease. J Comput Assist Tomogr 2000; 24: 614-619.

31. Natalin RA, Prince MR, Grossman ME, Silvers D, Landman J. Contemporary applications and limitations of magnetic resonance imaging contrast materials. J Urol 2010; 183(1): 27-33.

32. Bae KT, Tao C, Zhu F, Bost JE, Chapman AB, Grantham
JJ, Torres VE, Guay-Woodford LM, Meyers CM, Bennett WM and Consortium for Radiologic Imaging Studies of Polycystic Kidney Disease (CRISP). MRI-based Kidney Volume Measurements in ADPKD: Reliability and Effect of Gadolinium Enhancement. Clin J Am Soc Nephrol 2009; 4: 719-725.

33. Grantham JJ, Cook LT, Torres VE, Bost JE, Chapman AB, Harris PC, Guay-Woodford LM, Bae KT, for the CRISP Investigators. Determinants of renal volume in autosomal dominant polycystic kidney disease. Kidney Int 2008; 73 : 108-116.

34. Mignani R, Corsi C, De Marco M, Caiani EG, Santucci G, Cavagna E, Severi S, Cagnoli L. Assessment of Kidney Volume in Polycystic Kidney Disease Using Magnetic Resonance Imaging without Contrast Medium. Am J Nephrol 2011;33:176-184.

35. Ruggenenti P, Remuzzi A, Ondei P, Fasolini G, Antiga L, Ene-Iordache B, Remuzzi G, Epstein FH. Safety and efficacy of long-acting somatostatin treatment in autosomal-dominant polycystic kidney disease.Kidney Int 2005 Jul;68(1):206-16.

36. Perico N, Antiga L, Caroli A, Ruggenenti P, Fasolini G, Cafaro M, Ondei P, Rubis N, Diadei O, Gherardi G, Prandini S, Panozo A, Bravo RF, Carminati S, De Leon FR, Gaspari F, Cortinovis M, Motterlini N, Ene-Iordache B, Remuzzi A, Remuzzi G. Sirolimus therapy to halt the progression of ADPKD.J Am Soc Nephrol 2010 Jun;21(6):1031-40.

37. Serra AL, Poster D, Kistler AD, Krauer F, Raina S, Young J, Rentsch KM, Spanaus KS, Senn O, Kristanto P, Scheffel H, Weishaupt D, and Wüthrich RP. Sirolimus and Kidney Growth in Autosomal Dominant Polycystic Kidney Disease. New Eng J Med 2010; 363(9):820-829.

38. Walz G, Budde K, Mannaa N, Nürnberger J, Wanner C, Sommerer C, Kunzendorf U, Banas B, Hörl WH, Obermüller N, Arns W, Pavenstädt H, Gaedeke J, Büchert M, May C, Gschaidmeier H, Kramer S, Eckardt K. Everolimus in Patients with Autosomal Dominant Polycystic Kidney Disease. New Eng J Med 2010; 363(9):830-840.

39. Watnick T and Germino GG. mTOR Inhibitors in Polycystic Kidney Disease. New Eng J Med 2010; 363(9):879-881.

40. Grantham JJ, Bennett WM, Perrone RD. mTOR inhibitors and autosomal dominant polycystic kidney disease. N Engl J Med. 2011;364(3):286-7.

41. Irazabal MV, Torres VE, Hogan MC, Glockner J, King BF, Ofstie TG, Krasa HB, Ouyang J, Czerwiec FS. Short-term effects of tolvaptan on renal function and volume in patients with Autosomal Dominant Polycystic Kidney Disease. Kidney Int 2011, on-line 4 maggio 2011. 